\title{
ON JOINT SPECTRUM OF INFINITE DIRECT SUMS
}

\section{Shuilin Jin, Qinghua Jiang, Yadong WANG AND GuAngREn DuAN}

Abstract. For families of uniformly bounded $n$-tuples $T_{k}=\left(T_{k}^{1}, \cdots, T_{k}^{n}\right), k=1,2, \cdots$ of commuting operators on $\mathscr{H}$, the joint spectrum of $\oplus_{k=1}^{\infty} T_{k}$ is considered.

Mathematics subject classification (2010): Primary 47A13.

Keywords and phrases: Joint spectrum, joint point spectrum.

\section{REFERENCES}

[1] R. CURTO, Fredholm and invertible n-tuples of operators, Trans. Amer. Math. Soc. 266 (1981), 129159.

[2] R. CuRto And K. Yan, The Taylor spectrum of infinite direct sums, Contemp. Math. 120 (1991), $19-27$.

[3] M. Сhō And M. TAKAguchi, Joint spectra of matrices, Sci. Rep. Hirosaki Univ. 26 (1979), 15-19.

[4] J. PushPa AND S. M. PATEL, On joint spectrum, joint numerical range and joint spectral radius, Glasnik Mat. Ser. III 13 (1977), 315-321.

[5] J. L. TAYLOR, A joint spectrum for several commuting operators, J. Funct. Anal. 6 (1970), 172-191.

[6] J. L. TAYLOR, The analytic functional calculus for several commuting operators, Acta Math. 125 (1970), 1-38. 